PROCEEDINGS OF THE

AMERICAN MATHEMATICAL SOCIETY

Volume 132 , Number 12, Pages 3525-3536

S 0002-9939(04)07554-9

Article electronically published on July 12, 2004

\title{
HARDY'S INEQUALITIES FOR HERMITE AND LAGUERRE EXPANSIONS
}

\author{
R. RADHA AND S. THANGAVELU \\ (Communicated by Andreas Seeger)
}

\begin{abstract}
Hardy's inequalities are proved for higher-dimensional Hermite and special Hermite expansions of functions in Hardy spaces. Inequalities for multiple Laguerre expansions are also deduced.
\end{abstract}

\section{INTRODUCTION}

A well-known result of Paley states that if $f \in L^{p}(T), 1<p \leq 2$ and if $f \sim$ $\sum c_{k} e^{i k \theta}$, then

$$
\sum_{k=-\infty}^{\infty} \frac{\left|c_{k}\right|^{p}}{(1+|k|)^{2-p}} \leq c\|f\|_{p}^{p}
$$

This result fails when $p=1$, but Hardy proved that if $f \in \operatorname{Re} H^{1}$, then the inequality

$$
\sum_{k=-\infty}^{\infty} \frac{\left|c_{k}\right|}{(1+|k|)} \leq c\|f\|_{H^{1}}
$$

holds; here $H^{1}$ is the Hardy space. Later Hardy and Littlewood established a similar inequality for all $f \in H^{p}, 0<p \leq 1$.

Analogues of Hardy's equality in the context of eigenfunction expansions have been considered by several authors. In [1] Colzani-Travaglini established a Hardy inequality for eigenfunction expansions associated to the Laplace-Beltrami operator on compact Riemannian manifolds. They have also treated compact symmetric spaces. Kanjin 2 proved Hardy's inequality for the one-dimensional Hermite and Laguerre expansions of functions $f$ from $H^{1}(\mathbb{R})$. Later, Satake [5] treated $H^{p}(\mathbb{R}), 0<p \leq 1$ in the case of Laguerre expansions.

Let us briefly recall the inequalities proved by Kanjin and Satake. Let $h_{k}, k=$ $0,1,2, \cdots$ be the normalized Hermite functions on $\mathbb{R}$. For $f \in H^{1}(\mathbb{R})$, Kanjin proved that

$$
\sum_{k=0}^{\infty} \frac{\left|<f, h_{k}>\right|}{(1+k)^{29 / 36}} \leq C\|f\|_{H^{1}} .
$$

Received by the editors February 18, 2002 and, in revised form, October 14, 2002.

2000 Mathematics Subject Classification. Primary 42C10; Secondary 42B30, 42C05, 33 C45.

Key words and phrases. Hardy spaces, Hermite, special Hermite and Laguerre functions, twisted convolution. 
If $L_{k}^{\alpha}$ stands for Laguerre functions of type $\alpha>-1$ and if $f \in H^{p}\left(\mathbb{R}^{+}\right), 0<p \leq 1$, Satake proved the inequality

$$
\sum_{k=0}^{\infty} \frac{\left|<f, L_{k}^{\alpha}>\right|^{p}}{(1+k)^{2-p}} \leq C\|f\|_{H^{p}\left(\mathbb{R}^{+}\right)}^{p} .
$$

In studying regularity properties of spherical means on $\mathbf{C}^{n}$, the second author 7] has proved Hardy's inequality for special Hermite expansions. The first author [4] has treated the higher-dimensional Hermite expansions.

The aim of this paper is to establish inequalities of Hardy type for higherdimensional Hermite and special Hermite expansions. We also deduce Hardy inequalities for multiple Laguerre expansions.

The authors wish to thank the referee for meticulously reading the manuscript and pointing out several typographical errors.

\section{HeRmite EXPANSIONS}

The Hermite polynomials $H_{k}(x), k=0,1,2, \cdots$ are defined by

$$
H_{k}(x)=(-1)^{k}\left(\frac{d}{d x}\right)^{k}\left(e^{-x^{2}}\right) e^{x^{2}} .
$$

The normalized Hermite functions $h_{k}$ are then defined by

$$
H_{k}(x)=\left(2^{k} \sqrt{\pi} k !\right)^{-\frac{1}{2}} H_{k}(x) e^{-\frac{1}{2} x^{2}} .
$$

Then $\left\{h_{k}: k=0,1,2, \cdots\right\}$ forms an orthonormal basis for $L^{2}(\mathbb{R})$. The $n$-dimensional Hermite functions $\Phi_{\alpha}(x), x \in \mathbb{R}^{n}, \alpha \in \mathbb{N}^{n}$ are defined by taking tensor products of one-dimensional functions:

$$
\Phi_{\alpha}(x)=\prod_{j=1}^{n} h_{\alpha_{j}}\left(x_{j}\right) .
$$

Then it is clear that $\left\{\Phi_{\alpha}: \alpha \in \mathbb{N}^{n}\right\}$ is an orthonormal basis for $L^{2}\left(\mathbb{R}^{n}\right)$.

The Hermite expansion of a function $f \in L^{2}\left(\mathbb{R}^{n}\right)$ is written as

$$
f \sim \sum_{\alpha} \hat{f}(\alpha) \Phi_{\alpha}
$$

where the Fourier-Hermite coefficients $\hat{f}(\alpha)$ are given by

$$
\hat{f}(\alpha)=\int_{\mathbb{R}^{n}} f(x) \Phi_{\alpha}(x) d x .
$$

The functions $\Phi_{\alpha}$ are eigenfunctions of the operator $H=-\Delta+|x|^{2}$ with eigenvalues $(2|\alpha|+n)$ where $|\alpha|=\alpha_{1}+\alpha_{2}+\cdots+\alpha_{n}$. If we let

$$
P_{k} f=\sum_{|\alpha|=k} \hat{f}(\alpha) \Phi_{\alpha}
$$

stand for the projection of $f$ onto the $k$ th eigenspace of the operator $H$, then the Hermite expansion of $f$ takes the form

$$
f \sim \sum_{k=0}^{\infty} P_{k} f .
$$


The series converges in $L^{2}\left(\mathbb{R}^{n}\right)$, and we have the Plancherel formula

$$
\|f\|_{2}^{2}=\sum_{k=0}^{\infty}\left\|P_{k} f\right\|_{2}^{2}=\sum_{\alpha}|\hat{f}(\alpha)|^{2} .
$$

The Hardy spaces $H^{p}\left(\mathbb{R}^{n}\right), 0<p \leq 1$ can be defined in several ways. Let $p_{t}(x)=(4 \pi t)^{-\frac{n}{2}} e^{-\frac{|x|^{2}}{4 t}}$ be the heat kernel associated to the Laplacian $\Delta$ on $\mathbb{R}^{n}$. Then $H^{p}\left(\mathbb{R}^{n}\right)$ is defined to be the space of tempered distributions for which

$$
\|f\|_{H^{p}}^{p}=\int_{\mathbb{R}^{n}}\left(\sup _{t>0}\left|f * p_{t}(x)\right|\right)^{p} d x
$$

is finite. Every $f \in H^{p}\left(\mathbb{R}^{n}\right)$ has an atomic decomposition. Recall that a function $a(x)$ is called an $H^{p}$ atom if (i) $a$ is supported in a ball $B$; (ii) $|a(x)| \leq|B|^{-\frac{1}{p}}$ a.e. and (iii) $\int x^{\alpha} a(x) d x=0$ for all $\alpha$ with $|\alpha| \leq n\left(\frac{1}{p}-1\right)$. Then it is well known that every $f \in H^{p}\left(\mathbb{R}^{n}\right)$ has a decomposition

$$
f(x)=\sum_{k} \lambda_{k} a_{k}(x)
$$

where the $a_{k}$ are $H^{p}$ atoms. Moreover,

$$
C_{1}\|f\|_{H^{p}}^{p} \leq \sum_{k}\left|\lambda_{k}\right|^{p} \leq C_{2}\|f\|_{H^{p}}^{p}
$$

We make use of this atomic decomposition in the proof of the following Hardy's inequality.

Theorem 2.1. Let $n \geq 2,0<p \leq 1$. Then there exists a constant $C>0$ such that

$$
\sum_{k=0}^{\infty}\left\|P_{k} f\right\|_{2}^{p}(2 k+n)^{-\sigma} \leq C\|f\|_{H^{p}}^{p}
$$

for all $f \in H^{p}\left(\mathbb{R}^{n}\right)$ where $\sigma=\left(\frac{n}{2}+1\right)\left(\frac{2-p}{2}\right)$.

The proof of this theorem is based on the atomic decomposition of $H^{p}\left(\mathbb{R}^{n}\right)$ and the following estimate.

Lemma 2.2. Let $n \geq 2$. Then for all $\alpha \in \mathbb{N}^{n}$,

$$
\sup _{x \in \mathbb{R}^{n}} \sum_{|\mu|=k}\left|\partial^{\alpha} \Phi_{\mu}(x)\right|^{2} \leq C_{\alpha} k^{\frac{n}{2}+|\alpha|-1} .
$$

When $\alpha=0$ the lemma has been proved in [8] (see Lemma 3.2.2). Assuming the lemma for a moment, we first complete the proof of the theorem.

Every $f \in H^{p}$ has an atomic decomposition

$$
f(x)=\sum_{j=0}^{\infty} \lambda_{j} a_{j}(x)
$$

and since the $P_{k}$ are continuous on the space of tempered distributions, we have

$$
P_{k} f(x)=\sum_{j=0}^{\infty} \lambda_{j} P_{k} a_{j}(x) .
$$


In view of this it is enough to show that

$$
\sum_{k=0}^{\infty}\left\|P_{k} f\right\|_{2}^{p}(2 k+n)^{-\sigma} \leq C
$$

for all $H^{p}$ atoms $f$, with $C$ independent of $f$. Let $f$ be such an atom supported in the ball $B=B(z, r)$. Let

$$
\Phi_{k}(x, y)=\sum_{|\mu|=k} \Phi_{\mu}(x) \Phi_{\mu}(y)
$$

be the kernel of $P_{k}$ so that

$$
P_{k} f(x)=\int_{B} f(y) \Phi_{k}(x, y) d y .
$$

Taylor expanding the function $F(t)=\Phi_{k}(x, z+t(y-z))$ about $t=0$ we have

$$
\Phi_{k}(x, y)=\sum_{k=0}^{N} \frac{\partial_{t}^{k} F(0)}{k !}+\frac{1}{(N+1) !} \partial_{t}^{N+1} F(s)
$$

where $0<s<1$. If $N$ is the integral part of $n\left(\frac{1}{p}-1\right)$, then for $0 \leq k \leq N, \partial_{t}^{k} F(0)$ involves polynomials of the form $(y-z)^{\alpha},|\alpha| \leq N$ and hence

$$
P_{k} f(x)=\left.\frac{1}{(N+1) !} \partial_{t}^{N+1} \Phi_{k}(x, z+t(y-z))\right|_{t=s} .
$$

Thus $P_{k} f(x)$ is a finite linear combination of terms of the form

$$
\int_{|y-z| \leq r} f(y) \partial_{y}^{\alpha} \Phi_{k}(x, z+s(y-z))(y-z)^{\alpha} d y
$$

where $|\alpha|=N+1$.

By Minkowski's integral inequality, the $L^{2}$ norm of the above integral is bounded by

$$
\int_{|y-z| \leq r} f(y)|y-z|^{N+1}\left\|\partial_{y}^{\alpha} \Phi_{k}(., z+s(y-z))\right\|_{2} d y
$$

(with respect to $x$ ).

Since

$$
\left\|\partial_{y}^{\alpha} \Phi_{k}(\cdot, z+s(y-z))\right\|_{2}^{2}=\sum_{|\mu|=k}\left(\partial_{y}^{\alpha} \Phi_{\mu}(z+s(y-z))^{2},\right.
$$

using the estimate of the lemma we get

$$
\left\|\partial_{y}^{\alpha} \Phi_{k}(\cdot, z+s(y-z))\right\|_{2}^{2} \leq C(2 k+n)^{\frac{n}{2}+N} .
$$

Therefore, we have the estimate

$$
\begin{aligned}
\left\|P_{k} f\right\|_{2} & \leq C r^{N+1}(2 k+n)^{\frac{n}{4}+\frac{N}{2}} \int_{|y-z| \leq r}|f(y)| d y \\
& \leq C(2 k+n)^{\frac{n}{4}+\frac{N}{2}} r^{N+1-\frac{n}{p}+n} .
\end{aligned}
$$

Having estimated $\left\|P_{k} f\right\|_{2}$ consider now

$$
\sum_{k=0}^{\infty}\left\|P_{k} f\right\|_{2}^{p}(2 k+n)^{-\sigma}=S_{1}+S_{2}
$$


where the first sum is

$$
S_{1}=\sum_{(2 k+n) \leq r^{-2}}\left\|P_{k} f\right\|_{2}^{p}(2 k+n)^{-\sigma}
$$

which is bounded by

$$
\begin{gathered}
C r^{(N+1) p-n+n p} \sum_{(2 K+n) \leq r^{-2}}(2 k+n)^{-\sigma+\left(\frac{n}{4}+\frac{N}{2}\right) p} \\
\leq C r^{(N+1) p-n+n p} r^{2 \sigma-\left(\frac{n}{2}+N\right) p-2}=C
\end{gathered}
$$

since $2 \sigma=\left(\frac{n}{2}+1\right)(2-p)$. On the other hand,

$$
\begin{aligned}
S_{2} & =\sum_{(2 k+n)>r^{-2}}\left\|P_{k} f\right\|_{2}^{p}(2 k+n)^{-\sigma} \\
& \leq\left(\sum_{k=0}^{\infty}\left\|P_{k} f\right\|_{2}^{2}\right)^{\frac{p}{2}}\left(\sum_{(2 k+n)>r^{-2}}(2 k+n)^{-\frac{2 \sigma}{2-p}}\right)^{\frac{2-p}{2}} \\
& \leq\|f\|_{2}^{p} r^{-2\left(1-\frac{2 \sigma}{2-p}\right)\left(\frac{2-p}{2}\right)} \\
& \leq C r^{-n+\frac{n p}{2}} r^{2 \sigma+p-2}=C .
\end{aligned}
$$

Thus we have obtained the estimate

$$
\sum_{k=0}^{\infty}\left\|P_{k} f\right\|_{2}^{p}(2 k+n)^{-\sigma} \leq C
$$

where $C$ is independent of $f$. This completes the proof of the theorem.

We now prove the estimate stated in Lemma 2.2. We prove the lemma using induction on the dimension. We first consider the case $n=2$. Writing $x=(t, s), \alpha=\left(\alpha_{1}, \alpha_{2}\right)$ we consider

$$
\sum_{|\mu|=k}\left|\partial^{\alpha} \Phi_{\mu}(x)\right|^{2}=\sum_{j=0}^{k}\left|\partial_{t}^{\alpha_{1}} h_{j}(t)\right|^{2}\left|\partial_{s}^{\alpha_{2}} h_{k-j}(s)\right|^{2} .
$$

The Hermite functions satisfy the relations

$$
\left(-\frac{\partial}{\partial t}+t\right) h_{j}(t)=(2 j+2)^{\frac{1}{2}} h_{j+1}(t)
$$

and

$$
\left(\frac{\partial}{\partial t}+t\right) h_{j}(t)=(2 j)^{\frac{1}{2}} h_{j-1}(t)
$$

where $h_{-1}(t)=0$. Writing

$$
-\frac{\partial}{\partial t}=\frac{1}{2}\left(-\frac{\partial}{\partial t}+t\right)-\frac{1}{2}\left(\frac{\partial}{\partial t}+t\right)
$$

and using the above formulas, we get

$$
-\frac{\partial}{\partial t} h_{j}(t)=\frac{1}{2}(2 j+2)^{\frac{1}{2}} h_{j+1}(t)-\frac{1}{2}(2 j)^{\frac{1}{2}} h_{j-1}(t) .
$$


In view of this, considering $k \geq|\alpha|$, iterations show that $\sum_{|\mu|=k}\left|\partial^{\alpha} \Phi_{\mu}(x)\right|^{2}$ is bounded by a finite linear combination of terms of the form

$$
C(2 k+2)^{\alpha_{1}+\alpha_{2}} \sum_{j=0}^{k}\left|h_{j+l}(t)\right|^{2}\left|h_{k-j+m}(s)\right|^{2}
$$

where $l$ and $m$ are integers with $|l|,|m| \leq k$. The last sum is dominated by

$$
\sum_{j=0}^{k+m+l}\left|h_{j}(t)\right|^{2}\left|h_{k+m+l-j}(s)\right|^{2} \leq C k^{\frac{n}{2}-1}
$$

in view of the estimate (with $n=2$ )

$$
\sup _{x \in \mathbb{R}^{n}} \sum_{|\mu|=k}\left|\Phi_{\mu}(x)\right|^{2} \leq C k^{\frac{n}{2}-1}
$$

proved in Lemma 3.2.2 of [ [8]. Thus we have proved the lemma when $n=2$.

Assuming the estimate for the $n$-dimensional case, consider the sum in $(n+1)$ dimensions. Writing $x=(y, t), y \in \mathbb{R}^{n}, t \in \mathbb{R}$ and $\alpha=(\beta, l), \beta \in \mathbb{N}^{n}, l \in \mathbb{N}$ we have

$$
\begin{aligned}
\sum_{|\mu|=k}\left|\partial^{\alpha} \Phi_{\mu}(x)\right|^{2} & =\sum_{|\gamma|+j=k}\left|\partial_{y}^{\beta} \Phi_{\gamma}(y)\right|^{2}\left|\partial_{t}^{l} h_{j}(t)\right|^{2} \\
& =\sum_{j=0}^{k}\left(\sum_{|\gamma|=k-j}\left|\partial_{y}^{\beta} \Phi_{\gamma}(y)\right|^{2}\right)\left|\partial_{t}^{l} h_{j}(t)\right|^{2} .
\end{aligned}
$$

By the induction hypothesis the above is bounded by

$$
\begin{aligned}
& C \sum_{j=0}^{k}(k-j)^{\frac{n}{2}+|\beta|-1}\left|\partial_{t}^{l} h_{j}(t)\right|^{2} \\
& \leq C k^{\frac{n}{2}+|\beta|-1} \sum_{j=0}^{k}\left|\partial_{t}^{l} h_{j}(t)\right|^{2} .
\end{aligned}
$$

Using (2.1) the last term is easily seen to be bounded by terms of the form

$$
C k^{l} \sum_{j=0}^{k}\left|h_{j}(t)\right|^{2} \leq C k^{l+\frac{1}{2}}
$$

where we have used the estimate

$$
\sup _{t \in \mathbb{R}} \sum_{j=0}^{k}\left|h_{j}(t)\right|^{2} \leq C k^{\frac{1}{2}}
$$

proved in [8]; see Lemma (3.2.1). Finally, we have the estimate

$$
\sum_{|\mu|=k}\left|\partial^{\alpha} \Phi_{\mu}(x)\right|^{2} \leq C k^{\frac{n+1}{2}+|\beta|+l-1},
$$

which completes the induction since $|\beta|+l=|\alpha|$.

We conclude this section by proving another version of Hardy's inequality for Hermite expansions. 
Theorem 2.3. Let $n \geq 2$ and $0<p \leq 1$. Then there is a constant $C$ such that

$$
\sum_{\mu}|\hat{f}(\mu)|^{p}(2|\mu|+n)^{-\sigma} \leq C\|f\|_{H^{p}}^{p}
$$

for all $f \in H^{p}\left(\mathbb{R}^{n}\right)$ where $\sigma=\frac{3 n}{4}(2-p)$.

Proof. As before it is enough to consider $H^{p}$ atoms. If $f$ is such an atom supported in $B=B(z, r)$, then $\hat{f}(\mu)$ is a finite linear combination of terms of the form

$$
\int_{|y-z| \leq r} f(y)(y-z)^{\alpha} \partial_{y}^{\alpha} \Phi_{\mu}(z+s(y-z)) d y
$$

where $|\alpha|=N+1$. Therefore,

$$
|\hat{f}(\mu)| \leq C \sup _{x}\left|\partial^{\alpha} \Phi_{\mu}(x)\right|\|f\|_{2} r^{\frac{n}{2}+|\alpha|} .
$$

Since $\|f\|_{2}^{p} \leq C r^{-n+\frac{n p}{2}}$ we can rewrite the above as

$$
|\hat{f}(\mu)| \leq C \sup _{x}\left|\partial^{\alpha} \Phi_{\mu}(x)\right| r^{n\left(1-\frac{1}{p}\right)+N+1} .
$$

As before we split the sum into two parts and consider first

$$
S_{2}=\sum_{2|\mu|+n>r^{-2}}(2|\mu|+n)^{-\sigma}|\hat{f}(\mu)|^{p} .
$$

Applying Hölder's inequality, we get

$$
\begin{aligned}
S_{2} & \leq\|f\|_{2}^{p}\left(\sum_{2|\mu|+n>r^{-2}}(2|\mu|+n)^{-\frac{2 \sigma}{2-p}}\right)^{\frac{2-p}{2}} \\
& \leq\|f\|_{2}^{p}\left(\sum_{2 k+n>r^{-2}}(2 k+n)^{-\frac{2 \sigma}{2-p}+n-1}\right)^{\frac{2-p}{2}} .
\end{aligned}
$$

Since $\|f\|_{2}^{p} \leq C r^{-n+\frac{n}{2} p}$ and the last sum is bounded by $r^{2 \sigma-n(2-p)}=r^{n-\frac{n}{2} p}$ we get the estimate $S_{2} \leq C$.

Using the estimate for $|\hat{f}(\mu)|$ we see that

$$
S_{1} \leq C r^{n p\left(1-\frac{1}{p}\right)+(N+1) p} \sum_{2|\mu|+n \leq r^{-2}}(2|\mu|+n)^{-\sigma} \sup _{x}\left|\partial^{\alpha} \Phi_{\mu}(x)\right|^{p} .
$$

Now using the estimate proved in Lemma 2.2,

$$
\begin{aligned}
\sum_{|\mu|=k}\left|\partial^{\alpha} \Phi_{\mu}(x)\right|^{p} & \leq C k^{\frac{n-1}{2}(2-p)}\left(\sum_{|\mu|=k}\left|\partial^{\alpha} \Phi_{\mu}(x)^{2}\right|\right)^{\frac{p}{2}} \\
& \leq C k^{\frac{n-1}{2}(2-p)} k^{\left(\frac{n}{2}+N\right) \frac{p}{2}} .
\end{aligned}
$$

In view of this we obtain, after some simplification,

$$
\begin{aligned}
S_{1} & \leq C r^{n p\left(1-\frac{1}{p}\right)+(N+1) p} \sum_{(2 k+n) \leq r^{-2}}(2 k+n)^{\frac{p}{2}\left(N+1-n\left(\frac{1}{p}-1\right)\right)-1} \\
& \leq C r^{n p\left(1-\frac{1}{p}\right)+(N+1) p} r^{-p(N+1)+n p\left(\frac{1}{p}-1\right)}=C .
\end{aligned}
$$


Therefore, we have proved that

$$
\sum_{\mu}|\hat{f}(\mu)|^{p}(2|\mu|+n)^{-\sigma} \leq C
$$

whenever $f$ is an $H^{p}$ atom with $C$ independent of $f$. This completes the proof of Theorem 2.3.

\section{Special Hermite expansions}

We recall the definition and some basic properties of the special Hermite functions. We refer to 8 for more about these functions. For each $\alpha, \beta \in \mathbb{N}^{n}$ define

$$
\Phi_{\alpha \beta}(z)=(2 \pi)^{-\frac{n}{2}} \int_{\mathbb{R}^{n}} e^{i\left(x \xi+\frac{1}{2} x y\right)} \Phi_{\alpha}(\xi+y) \Phi_{\beta}(\xi) d \xi
$$

where $z=x+i y \in \mathbf{C}^{n}$. Then $\left\{\Phi_{\alpha, \beta}: \alpha, \beta \in \mathbb{N}^{n}\right\}$ forms an orthonormal basis for $L^{2}\left(\mathbf{C}^{n}\right)$. The special Hermite expansion for $f \in L^{2}\left(\mathbf{C}^{n}\right)$ is given by

$$
f \sim \sum_{\alpha, \beta}\left\langle f, \Phi_{\alpha, \beta}\right\rangle \Phi_{\alpha \beta} .
$$

The functions $\Phi_{\alpha \beta}$ are eigenfunctions of the operator

$$
L=-\Delta_{z}+\frac{1}{4}|z|^{2}-i \sum_{j=1}^{n}\left(x_{j} \frac{\partial}{\partial y_{j}}-y_{j} \frac{\partial}{\partial x_{j}}\right)
$$

with eigenvalues $(2|\beta|+n)$. If we let

$$
\varphi_{k}(z)=L_{k}^{n-1}\left(\frac{1}{2}|z|^{2}\right) e^{-\frac{1}{4}|z|^{2}}
$$

stand for Laguerre functions of type $(n-1)$, then the special Hermite expansion takes the form

$$
f(z)=(2 \pi)^{-n} \sum_{k=0}^{\infty} f \times \varphi_{k}(z)
$$

where the twisted convolution $f \times \varphi_{k}(z)$ is defined by

$$
f \times \varphi_{k}(z)=\int_{\mathbf{C}^{n}} f(z-w) e^{\frac{i}{2} \operatorname{Im}(z \cdot \bar{w})} \varphi_{k}(w) d w .
$$

It can be shown that $(2 \pi)^{-n} f \times \varphi_{k}(z)$ is the projection of $f$ onto the $k$ th eigenspace of $L$.

If $p_{t}(z)$ stands for the heat kernel associated to $L$, then the twisted Hardy spaces $\mathcal{H}^{p}, 0<p \leq 1$ can be defined as the spaces of tempered distributions for which

$$
\sup _{t>0}\left|f \times \varphi_{k}(z)\right| \in L^{p}\left(\mathbf{C}^{n}\right) .
$$

When $p=1$ this space has been studied in [3] and there is an atomic decomposition available for $f \in \mathcal{H}^{1}\left(\mathbf{C}^{n}\right)$. More generally, an $\mathcal{H}^{p}$ atom is a function $f$ supported in a ball $B_{r}(w)$ satisfying the estimate $|f(z)| \leq\left|B_{r}(w)\right|^{-\frac{1}{p}}$ and when $r \leq 1$, also satisfying the cancellation conditions

$$
\int_{B_{r}(w)} f(z)(z-w)^{\alpha} e^{\frac{i}{2} \operatorname{Im} z \cdot \bar{w}} d z=0
$$


for $|\alpha| \leq N, N$ being the integral part of $2 n\left(\frac{1}{p}-1\right)$. When $r>1$ no cancellation condition is assumed. We can define the atomic twisted Hardy space $\mathcal{H}_{a}^{p}$ as the space of all tempered distributions $f$ that can be represented as

$$
f=\sum_{j=0}^{\infty} \lambda_{j} a_{j}
$$

the series being convergent in the space of tempered distributions, where the $a_{j}$ are $\mathcal{H}^{p}$ atoms and $\sum_{j=0}^{\infty}\left|\lambda_{j}\right|^{p}<\infty$. The space $\mathcal{H}_{a}^{p}$ can be made into a metric space by means of the quasi-norm defined by

$$
\|f\|_{\mathcal{H}_{a}^{p}}^{p}=\inf \left\{\sum_{j=0}^{\infty}\left|\lambda_{j}\right|^{p}: f=\sum_{j=0}^{\infty} \lambda_{j} a_{j}\right\} .
$$

For $p=1$ it is known that $\mathcal{H}_{a}^{p}=\mathcal{H}^{p}$. However, this is not known for $0<p<1$. For special Hermite functions we have the following Hardy's inequality.

Theorem 3.1. Let $0<p \leq 1$. Then there is a constant $C$ such that

$$
\sum_{k=0}^{\infty}\left\|f \times \varphi_{k}\right\|_{2}^{p}(2 k+n)^{-\sigma} \leq C\|f\|_{\mathcal{H}_{a}^{p}}^{p}
$$

for all $f \in \mathcal{H}_{a}^{p}$ where $\sigma=\left(\frac{n+1}{2}\right)(2-p)$.

When $p=1$ this result has been proved in [7]. The key point is the estimate

$$
\sup _{z} \sum_{j=1}^{n}\left|\frac{\partial}{\partial z_{j}} \varphi_{k}(z)\right| \leq C k^{n},
$$

which easily follows from estimates on Laguerre functions. In the present case, we need estimates of the form

$$
\sup _{z}\left|\partial_{z}^{\alpha} \varphi_{k}(z)\right| \leq C k^{n+|\alpha|-1} .
$$

These estimates can be proved easily using properties of Laguerre functions. Once we have the above estimates we can proceed as in Theorem 2.1 (or as in [7) to prove Theorem 3.1. We leave the details to the interested reader.

The above theorem is proved for functions coming from the twisted Hardy spaces. There is an analogue of Theorem 2.1 for special Hermite expansions for functions from the ordinary Hardy spaces $H^{p}\left(\mathbf{C}^{n}\right)=H^{p}\left(\mathbb{R}^{2 n}\right)$. The functions $\Phi_{\alpha \beta}$ are also eigenfunctions of the operator $-\Delta+\frac{1}{4}|z|^{2}$ on $\mathbf{C}^{n}$ with eigenvalues $(|\alpha|+|\beta|+n)$, and hence we think of (3.1) as an eigenfunction expansion associated to $\left(-\Delta+\frac{1}{4}|z|^{2}\right)$, which is nothing but a scaled Hermite operator on $\mathbb{R}^{2 n}$.

Let $\hat{f}(\mu, \gamma)$ be the special Hermite coefficients of $f$ defined by

$$
\hat{f}(\mu, \gamma)=\int_{\mathbf{C}^{n}} f(z) \bar{\Phi}_{\mu \gamma}(z) d z .
$$

We prove the following result.

Theorem 3.2. Let $0<p \leq 1$. Then there is a constant $C$ such that

$$
\sum_{\mu, \gamma}|\hat{f}(\mu, \gamma)|^{p}(|\mu|+|\gamma|+n)^{-\sigma} \leq C\|f\|_{H^{p}}^{p}
$$

for all $f \in H^{p}\left(\mathbf{C}^{n}\right)$ where $\sigma=\frac{3 n}{2}(2-p)$. 
Again the proof uses atomic decomposition of $H^{p}\left(\mathbb{R}^{2 n}\right)$ and some estimates on the derivatives of the functions $\Phi_{\mu \gamma}(z)$. More precisely we need

$$
\sum_{|\mu|+|\gamma|=k}\left|\partial_{z}^{\alpha} \Phi_{\mu \gamma}(z)\right|^{2} \leq C k^{n+|\alpha|-1}
$$

where $\alpha \in \mathbb{N}^{2 n}$. This can be deduced from Lemma 2.2. As we remarked earlier, $\Phi_{\mu \gamma}(z)=\Phi_{\mu \gamma}(x, y)$ are eigenfunctions of the operator $\left(-\Delta+\frac{1}{4}\left(|x|^{2}+|y|^{2}\right)\right)$ on $\mathbb{R}^{2 n}$ with eigenvalues $(|\mu|+|\gamma|+n)$. On the other hand, the functions

$$
2^{\frac{n}{2}} \Phi_{\mu}\left(\frac{1}{\sqrt{2}} x\right) \Phi_{\gamma}\left(\frac{1}{\sqrt{2}} y\right)
$$

are also eigenfunctions of the the same operator with the same eigenvalues. Therefore, with $z=x+i y, w=u+i v$,

$$
\sum_{|\mu|+|\gamma|=k} \bar{\Phi}_{\mu \gamma}(w) \Phi_{\mu \gamma}(z)=2^{n} \sum_{|\mu|+|\gamma|=k} \Phi_{\mu}\left(\frac{1}{\sqrt{2}} x\right) \Phi_{\gamma}\left(\frac{1}{\sqrt{2}} y\right) \Phi_{\mu}\left(\frac{1}{\sqrt{2}} u\right) \Phi_{\gamma}\left(\frac{1}{\sqrt{2}} v\right)
$$

since both sides represent the kernel of the projection onto the $k$ th eigenspace. From this expression it is clear that the estimate (3.2) follows from Lemma 2.2.

Once we have the estimate (3.2) we can proceed as in Theorem 2.1 to complete the proof. We have stated Theorems 3.1 and 3.2 because of their roles in establishing Hardy inequalities for multiple Laguerre expansions. This is done in the next section.

\section{LAGUERRE EXPANSIONS}

Laguerre functions of type $\delta>-1$ are defined by the equation

$$
L_{k}^{\delta}(t) t^{\delta} e^{-t}=\frac{1}{k !}\left(\frac{d}{d t}\right)^{k}\left(e^{-t} t^{k+\delta}\right) .
$$

The normalized functions

$$
\Psi_{k}^{\delta}(t)=\left(\frac{\left.\Gamma(k+1) 2^{-\delta}\right)}{\Gamma(k+\delta+1)}\right)^{\frac{1}{2}} L_{k}^{\delta}\left(\frac{t^{2}}{2}\right) e^{-\frac{1}{4} t^{2}}
$$

form an orthonormal basis for $L^{2}\left(\mathbb{R}^{+}, t^{2 \delta+1} d t\right)$. When $f(z)=f(|z|)$ is a radial function on $\mathbf{C}^{n}$ it can be shown that

$$
(2 \pi)^{-n} f \times \varphi_{k}(z)=\left(\int_{0}^{\infty} f(r) \Psi_{k}^{n-1}(r) r^{2 n-1} d r\right) \Psi_{k}^{n-1}(t)
$$

where $t=|z|$. Thus the special Hermite expansions of radial functions reduce to Laguerre expansions. In view of this remark the following theorem follows from Theorem 3.1.

Theorem 4.1. Let $0<p \leq 1$. Then with $\sigma=\frac{n+1}{2}(2-p)$,

$$
\sum_{k=0}^{\infty}\left|<f, \Psi_{k}^{n-1}>\right|^{p}(2 k+n)^{-\sigma} \leq C\|f\|_{\mathcal{H}_{a}^{p}}^{p}
$$

for all functions $f$ for which $f(z)=f(|z|)$ belongs to $\mathcal{H}_{a}^{p}\left(\mathbf{C}^{n}\right)$. 
Our next result is about multiple Laguerre expansions. For $t=\left(t_{1} \cdots t_{n}\right), t_{j} \geq 0$, define

$$
\Psi_{\mu}^{\delta}(t)=\prod_{j=1}^{n} \Psi_{\mu_{j}}^{\delta_{j}}\left(t_{j}\right), \quad \mu \in \mathbb{N}^{n}, \delta=\left(\delta_{1}, \delta_{2}, \ldots, \delta_{n}\right) .
$$

Then $\left\{\Psi_{\mu}^{\delta}: \mu \in \mathbb{N}^{n}\right\}$ is an orthonormal basis for $L^{2}\left(\left(\mathbb{R}^{+}\right)^{n}, w_{\delta}(t) d t\right), w_{\delta}(t)=$ $\prod_{j=1}^{n} t^{2 \delta_{j}+1}$.

The multiple Laguerre expansion of $f$ in this space is given by

$$
f(t)=\sum_{\mu}\left\langle f, \Psi_{\mu}^{\delta}\right\rangle \Psi_{\mu}^{\delta}(t) .
$$

For $m \in \mathbb{N}^{n}$ let us denote by $H_{m}^{p}\left(\left(\mathbb{R}^{+}\right)^{n}\right)$ the space of all functions $f$ on $\left(\mathbb{R}^{+}\right)^{n}$ for which the associated function $F$ defined by

$$
F(z)=F\left(r_{1} e^{i \theta_{1}}, \ldots, r_{n} e^{i \theta_{n}}\right)=e^{i m \cdot \theta} f\left(r_{1}, r_{2}, \ldots, r_{n}\right)
$$

belongs to $\mathcal{H}_{a}^{p}\left(\mathbf{C}^{n}\right)$. Note that any such $F$ satisfies

$$
F\left(e^{i \varphi} z\right)=e^{i m \cdot \varphi} F(z), \quad \varphi \in \mathbb{R}^{n},
$$

and so they are called $m$-homogeneous.

For functions $f \in H_{m}^{p}$ we have the following Hardy's inequality.

Theorem 4.2. Let $0<p \leq 1$. Then there is a constant $C$ such that

$$
\sum_{\mu}\left|\left\langle f, \Psi_{\mu}^{m}\right\rangle\right|^{p}(2|\mu|+n)^{-\sigma} \leq C\|f\|_{H_{m}^{p}}^{p}
$$

for all $f \in H_{m}^{p}$ with $\sigma=\frac{3 n}{2}(2-p)$.

In the above theorem, $\|f\|_{H_{m}^{p}}$ stands for the $\|F\|_{H_{a}^{p}\left(\mathbf{C}^{n}\right)}$ of the associated function. We deduce the above theorem from Theorem 3.2. It can be checked, directly from the definition or from the explicit formula given in [8], that $\Phi_{\mu \gamma}(z)$ are $(\gamma-\mu)$ homogeneous. Therefore, integration in polar coordinates implies that when $F$ is $m$-homogeneous, then $\left(F, \Phi_{\mu \gamma}\right)=0$ unless $\gamma=\mu+m$, in which case

$$
\left\langle F, \Phi_{\mu, \mu+m}\right\rangle=\left\langle f, \Psi_{\mu}^{m}\right\rangle, \quad \mu \in \mathbb{N}^{n} .
$$

We refer to 8 for a proof of this. Once we have this it is clear that Theorem 4.2 is an immediate corollary of Theorem 3.2

\section{REFERENCES}

1. L. Colzani and G. Travaglini, Hardy-Lorentz spaces and expansions in eigenfunctions of the Laplace-Beltrami operator on compact manifolds, Colloq. Math. 58 (1990), 305 - 316. MR 92c:58142

2. Y. Kanjin, Hardy's inequalities for Hermite and Laguerre expansions, Bull. London Math. Soc. 29 (1997), 331 - 337. MR 98f:42024

3. G. Mauceri, M. A. Picardello and F. Ricci, A Hardy space associated with twisted convolution, Adv. Math. 39 (1981), 270 - 288. MR 83e:42026

4. R. Radha, Hardy type inequalities, Taiwanese J. Math 4 (2000), 447 - 456. MR 2002f:33013

5. M. Satake, Hardy's inequalities for Laguerre expansions, J. Math. Soc. Japan 52 (2000), 17 24. MR 2001c:42014

6. E. M. Stein, Harmonic Analysis, Princeton University Press, Princeton, NJ, 1993. MR 95c:42002 
7. S. Thangavelu, On regularity of twisted spherical means and special Hermite expansion, Proc. Indian Acad. Sci. Math. Sci. 103 (1993), 303 - 320. MR 95c:33015

8. S. Thangavelu, Lectures on Hermite and Laguerre expansions, Math. Notes, No. 42, Princeton University Press, Princeton, NJ, 1993. MR 94i:42001

School of Mathematics, Anna University, Chennai - 600 025, India

E-mail address: radharam@annauniv.edu

Current address: Department of Mathematics, Indian Institute of Technology, Chennai 600 036, India

Stat-Math Unit, Indian Statistical Institute, 8th Mile, Mysore Road (R.V. College Post), BANGalore - 560 059, India

E-mail address: veluma@isibang.ac.in 